\title{
The CCR4-NOT complex is a tumor suppressor in Drosophila melanogaster eye cancer models
}

\author{
Carmen Vicente ${ }^{1,2,6^{*}+}$, Rocco Stirparo ${ }^{1,2+}$, Sofie Demeyer ${ }^{1,2}$, Charles E. de Bock ${ }^{1,2}$, Olga Gielen ${ }^{1,2}$, Mardelle Atkins ${ }^{1,3}$,
} Jiekun Yan ${ }^{2,4}$, Georg Halder ${ }^{1,3}$, Bassem A. Hassan ${ }^{2,4,5}$ and Jan Cools ${ }^{1,2^{*}}$

\begin{abstract}
Background: The CNOT3 protein is a subunit of the CCR4-NOT complex, which is involved in mRNA degradation. We recently identified CNOT3 loss-of-function mutations in patients with T-cell acute lymphoblastic leukemia (T-ALL).

Methods: Here, we use different Drosophila melanogaster eye cancer models to study the potential tumor suppressor function of Not3, the CNOT3 orthologue, and other members of the CCR4-NOT complex.

Results: Our data show that knockdown of Not3, the structural components Not1/Not2, and the deadenylases twin/Pop2 all result in increased tumor formation. In addition, overexpression of Not3 could reduce tumor formation. Not3 downregulation has a mild but broad effect on gene expression and leads to increased levels of genes involved in DNA replication and ribosome biogenesis. $C y C B$ upregulation also contributes to the Not3 tumor phenotype. Similar findings were obtained in human T-ALL cell lines, pointing out the conserved function of Not3.
\end{abstract}

Conclusions: Together, our data establish a critical role for Not3 and the entire CCR4-NOT complex as tumor suppressor.

Keywords: CCR4-NOT, Leukemia, mRNA stability, Tumor suppressor, Drosophila melanogaster

\section{Background}

The CCR4-NOT complex is an essential and conserved multi-subunit complex that regulates gene expression [1]. Although it is implicated in many different cellular functions, it has been mainly studied for its mRNA deadenylation activity, a first step in mRNA degradation $[1,2]$. In humans, the CCR4-NOT complex consists of at least nine conserved "canonical" subunits: CNOT1, CNOT2, CNOT3, CNOT6, CNOT6L, CNOT7, CNOT8, CNOT9, and CNOT10. Among these subunits, CNOT6, CNOT6L, CNOT7, and CNOT8 have deadenylase activity and are directly responsible for the removal of the poly-A tail from the target mRNA [3].

Besides, other subunits of the complex are also important for mRNA degradation. For instance, deadenylation is suppressed by CNOT1 depletion and CNOT2 downregulation affects the length of mRNA poly-A tails

\footnotetext{
* Correspondence: cvicente@unav.es; jan.cools@kuleuven.be

${ }^{+}$Carmen Vicente and Rocco Stirparo contributed equally to this work

${ }^{1}$ Center for Cancer Biology, VIB, Leuven, Belgium

Full list of author information is available at the end of the article
}

$[4,5]$. Recent data indicate that CNOT3 is also involved in the control of mRNA stability. Cnot3 haplodeficiency in ob/ob mice ameliorated the obese phenotype through the regulation of the CCR4-NOT-mediated deadenylation of specific mRNAs involved in energy metabolism [6]. Furthermore, CNOT3 regulates bone mass through regulation of Rank mRNA stability [7]. Recently, it has been shown that CNOT3 contributes to early B cell development by controlling Igh rearrangement and $p 53$ mRNA stability [8].

Escape from post-transcriptional regulation of gene expression is a crucial step in the pathogenesis of cancer. Aberrant polyadenylation site usage, leading to a truncated 3'UTR, has been detected in many human malignancies and might allow malignant cells to escape regulation by both microRNA and RNA binding proteins $[9,10]$. The RNA-binding protein TTP has been shown to impair MYC-driven lymphoma development [11]. Deletion of the genes $Z f p 36 L 1$ and $Z f p 36 L 2$, which encode RNA-binding proteins, was shown to cause T-cell acute lymphoblastic

(c) The Author(s). 2018 Open Access This article is distributed under the terms of the Creative Commons Attribution 4.0 International License (http://creativecommons.org/licenses/by/4.0/), which permits unrestricted use, distribution, and reproduction in any medium, provided you give appropriate credit to the original author(s) and the source, provide a link to the Creative Commons license, and indicate if changes were made. The Creative Commons Public Domain Dedication waiver (http://creativecommons.org/publicdomain/zero/1.0/) applies to the data made available in this article, unless otherwise stated. 
leukemia (T-ALL) in mice due to impaired Notch1 mRNA degradation [12].

We recently identified loss-of-function mutations on the CNOT3 gene in patients with T-ALL [13]. Other studies have confirmed CNOT3 mutations in T-ALL and have also identified mutations in CNOT1 and CNOT2 [14-16]. Furthermore, T-ALL patients with HOXA-rearrangements and terminal 5q deletions show CNOT6 downregulation and high incidence of CNOT3 mutations [17]. These data suggest that the CCR4-NOT complex is involved in cancer, although it remains unclear how it is contributing to tumor development.

Here, we explored how loss of Not3, the CNOT3 orthologue, is involved in tumor development using loss-of-function and gain-of-function analyses in Drosophila melanogaster eye cancer models. We established that Not3 behaves as a tumor suppressor gene. Reduction of Not3 expression resulted in a significant increase in tumor incidence, while its overexpression suppressed tumor formation. Downregulation of other subunits of the CCR4-NOT complex also enhanced tumor formation. Our results indicate that the entire complex and its deadenylase activity are required for tumor suppression, which is linked with $C y c B$ upregulation and the regulation of genes implicated in DNA replication and ribosome biogenesis.

\section{Methods}

\section{Fly husbandry}

All crosses were raised on standard fly food at $25^{\circ} \mathrm{C}$. All fly lines used are listed in Additional file 1: Table S1. For the generation of eyeless $>$ UAS-RNAi Not3, eyeless $>$ UAS -Dl>UAS-RNAi Not3 and eyeless $>$ UAS-Dl $>$ UAS-RNAi twin animals standard methods were used to recombine UAS-RNAi Not3 (VDRC KK102144, v105990) or UAS-RNAi twin (VDRC KK108897, v104442) with an eyeless-Gal4 or an eyeless-Gal4>UAS-Dl insertion on the second chromosome. To perform experiments for quantifying proliferation, apoptosis, and differentiation, control crosses were established using eyeless $>$ UAS-Dl stock virgins and males carrying UAS-RNAi white. This chromosome was maintained in a stock balanced over CyO, GFP. Three independent crosses were established per experimental condition tested, and five transfers from each cross into new fly food flasks were performed.

\section{Analyses of eye tumor burden}

Adult animals of the correct genotypes were imaged using a Zeiss Apotome microscope. To analyze tumor burden, each eye was scored separately on flies with the genotype of interest (positive F1 progeny). The score of the eye tumor burden was performed double-blind (except for those experiments in which we investigated the function of the seven selected target genes, shown in Fig. 6). Eyes were counted as hyperplastic when showing at least one fold. Metastases were observed as masses of amorphous red-pigmented cells outside of the eye field (head, thorax, and abdomen). The percentages shown on the bar graphs represent the average percentage of three independent crosses, and the mean number of eyes analyzed is indicated on each graph ( $Y$-axis). In the experiments involving (1) the downregulation of Not3/twin on the sensitized, the Ras-V12, or the wild-type genetic backgrounds and (2) the overexpression of Not3 or the Not3 mutant construct on the eyeful genetic background, the whole eye area (region with presence of differentiated photoreceptors) was measured on representative adult eyes. Image processing and eye measurements were performed with ImageJ. Control eyes measured values were considered as $100 \%$. Values for eight individual discs were plotted. Data was analyzed using GraphPad Prism v6.

\section{Immunohistochemistry, imaging, and quantification}

Dissections and stainings were performed as previously described [18]. Primary antibodies used were rat anti-ELAV (1:100, DSHB), rabbit anti-Phospho-Histone-3 (1:1000, Millipore), and rabbit anti-cleaved-DCP1a (1:150, Cell signaling). All secondary antibodies were used at 1:500. Samples were mounted in Prolong antifade mounting media. Fluorescence imaging was performed using a Leica confocal microscope. Images were processed (Z-projection) using ImageJ/FIJI. Phospho-histone H3 (pH3), cleaved-DCP1a, and BrdU positive cells within the posterior part of eye discs from the appropriate genotypes (GFP negative larvae) were counted. Values for 10 individual discs were plotted. Data was graphed and analyzed using GraphPad Prism v6.

\section{RNA-sequencing sample preparation and analyses}

Dissection tools and surfaces were treated with RNAseq Away, and RNA was isolated using the RNAqueous-Micro Kit (Ambion). Eye-antennal imaginal discs were dissected from 30 L3 wandering larvae and transferred into RNAse-free ependorfs containing lysis buffer on ice. Following dissection, either RNA was extracted immediately or discs on lysis buffer were snap-frozen and kept at $-80^{\circ}$ $\mathrm{C}$ for later extraction. All the RNA samples showed high quality on the Bioanalyzer (Agilent Technologies). Next-generation sequencing libraries were constructed from 500 ng of total RNA using the Truseq RNA sample prep kit v2, and RNA-seq libraries were subjected to $1 \times$ 50 bp single-end sequencing on a HiSeq2500 instrument (Illumina). For each condition, three replicates were sequenced. The reads were cleaned with fastq-mcf, and a quality control was performed with FastQC (http:// www.bioinformatics.babraham.ac.uk/projects/fastqc). Reads were then mapped to the Drosophila melanogaster genome (dm6) with TopHat2 [19]. Subsequently, HTSeq-count [20] was used to count the number of reads per gene. For 
differential gene expression analysis, the Bioconductor package DESeq2 [21] was used. The lists of differential genes were then further analyzed using FlyMine [22].

\section{Drosophila Not3 overexpression and S2 cell transfection experiments}

All Not3 constructs were cloned into the pUASTattB vector. We used Canton S L3 wandering larval eye discs cDNA as template. Fragments were PCR amplified using the primers shown in Additional file 1: Table S2. All constructs were verified by Sanger sequencing. The transgenic flies were generated by BestGene (Strain \#9744, 89E11 acceptor site). Drosophila S2 cells were co-transfected with a pMT-Gal4 plasmid and a flag-tagged version of each of the UAS-Not3 plasmids. Gal4 expression was induced $24 \mathrm{~h}$ post-transfection by adding $\mathrm{CuSO}_{4}$. Cells were treated with cycloheximide $(50 \mu \mathrm{g} / \mathrm{ml}, 3 \mathrm{~h}$ treatment) $24 \mathrm{~h}$ after gene expression induction. Lysates were immunoblotted with anti-Flag antibodies. To test the stability of Cycb, fancl, or upd2 mRNAs in Drosophila S2 cells, co-transfections of the pMT-Gal4 plasmid and the RNAi plasmid targeting Not3 (construct ID 4068, dna4068 from the VDRC) or a UAS-YFP plasmid (as a negative control) were performed using an Amaxa nucleofector. Gal4 expression (thus, Not3 RNAi or YFP expression) was induced $24 \mathrm{~h}$ post-transfection by adding $\mathrm{CuSO} 4$. After $24 \mathrm{~h}$ of Not3 RNAi or YFP expression induction, transcription was stopped by addition of $5 \mu \mathrm{g} / \mathrm{ml}$ actinomycin D. Cells were harvested, and RNA was purified after $0,10,20,40$, 80 , and $160 \mathrm{~min}$ and quantified by qPCR. Three independent experiments were performed. Expression of the CG1239 gene was used as a control of no stabilization, since its expression was not significantly changed on our RNA-sequencing experiments after Not3 downregulation.

\section{CDNA synthesis and qPCR analyses}

cDNA synthesis from eye discs was performed using the QuantiTec Reverse Transcription kit following manual instructions. cDNA synthesis from S2 cells experiments was performed using the GoScript Reverse Transcription system protocol (Promega). Real-time qPCR reactions were performed using the GoTaq Real-Time kit, and reactions were run in a Lightcycler 480 device (Roche). Primers are listed in Additional file 1: Table S2.

\section{T-ALL cell lines electroporation and RNA stability assay}

Jurkat and CCRF-CEM cells were cultured under standard conditions. Electroporations were performed using the gene pulser $\mathrm{Xcell}^{\mathrm{lm}}$ electroporation system as previously described [23]. The negative control siRNA (D-001810-01-20), CNOT3 siRNA 326 (J-020319-06), and CNOT3 siRNA 328 (J-020319-08) were purchased from Dharmacon. Cells were treated with $5 \mu \mathrm{g} / \mathrm{ml}$ Actinomycin D (Sigma Aldrich) $24 \mathrm{~h}$ after electroporation.
RNA was immediately extracted at $0 \mathrm{~h}, 2 \mathrm{~h}$, and $4 \mathrm{~h}$ with Maxwell $^{\circ}$ simplyRNA Cells Kit. Regarding the RNA-sequencing analysis pipeline, reads were mapped to the human genome (GRCh37/hg19). For the differential gene expression analysis (DESeq2), a linear model was applied, as we needed to compare all conditions with each other (instead of a one-to-one comparison). The data was modeled as TP + KD + TP:KD, in which the term TP (time point) represents the mRNA degradation over time, the term $\mathrm{KD}$ (knockdown) is related to the knockdown of CNOT3, and the combined term TP:KD shows the impact of the CNOT3 knockdown on mRNA degradation. The differential genes resulting from this last term, which are the more stable mRNAs (less degraded), were then used for further analysis with DAVID [24].

\section{Results}

Not3 behaves as a tumor suppressor gene in Drosophila melanogaster eye cancer models

To investigate the tumor suppressor role of Not3, we downregulated or overexpressed it in various genetic backgrounds. As a first model, we used flies with overexpression of the Notch ligand Delta $(D l)$ in the eye (driven by $e y$-Gal4) which results in hyper-activation of the Notch signaling pathway, thereby, leading to an increase in eye size but no tumor development $[18,25]$. We refer to these flies as "sensitized" flies. We downregulated the expression of Not3 in this genetic background using three different UAS-RNAi Not3 lines, and a line with a P-element transposon insertion, in which the expression of one of the Not3 alleles has been shown to be disrupted [26]. Reduction of Not3 expression resulted in a remarkable increase in tumor incidence, from $7 \%$ of the eyes with control RNA interference (UAS-RNAi white) to up to $90 \%$ with the three different Not3 RNAi lines (Fig. 1a). Strikingly, inactivation of one allele of Not3 by the P-element insertion (which results in modest downregulation of Not3) was sufficient to induce tumor formation in $50 \%$ of the eyes (Fig. 1a). In all cases, tumor development was observed without metastases. These data support the hypothesis that loss of Not3 is sufficient to transform a sensitized lesion into a tumor, possibly by interfering with patterning and cell fate determination.

Next, we performed immunofluorescence stainings on sensitized L3 larval eye discs to investigate changes on the number of proliferative and differentiated cells upon Not3 downregulation. To do that, we used the phospho-Histone3 (pH3) and BrdU as markers of proliferation and ELAV as a marker of differentiation. Knockdown of Not3 expression was first confirmed by qPCR (Fig. 1b) and resulted in a significant increase of $\mathrm{pH} 3$ and BrdU positive cells (Fig. 1c). Downregulation of Not3 expression did not cause a significant loss of ELAV expressing cells (differentiated cells), although it did 


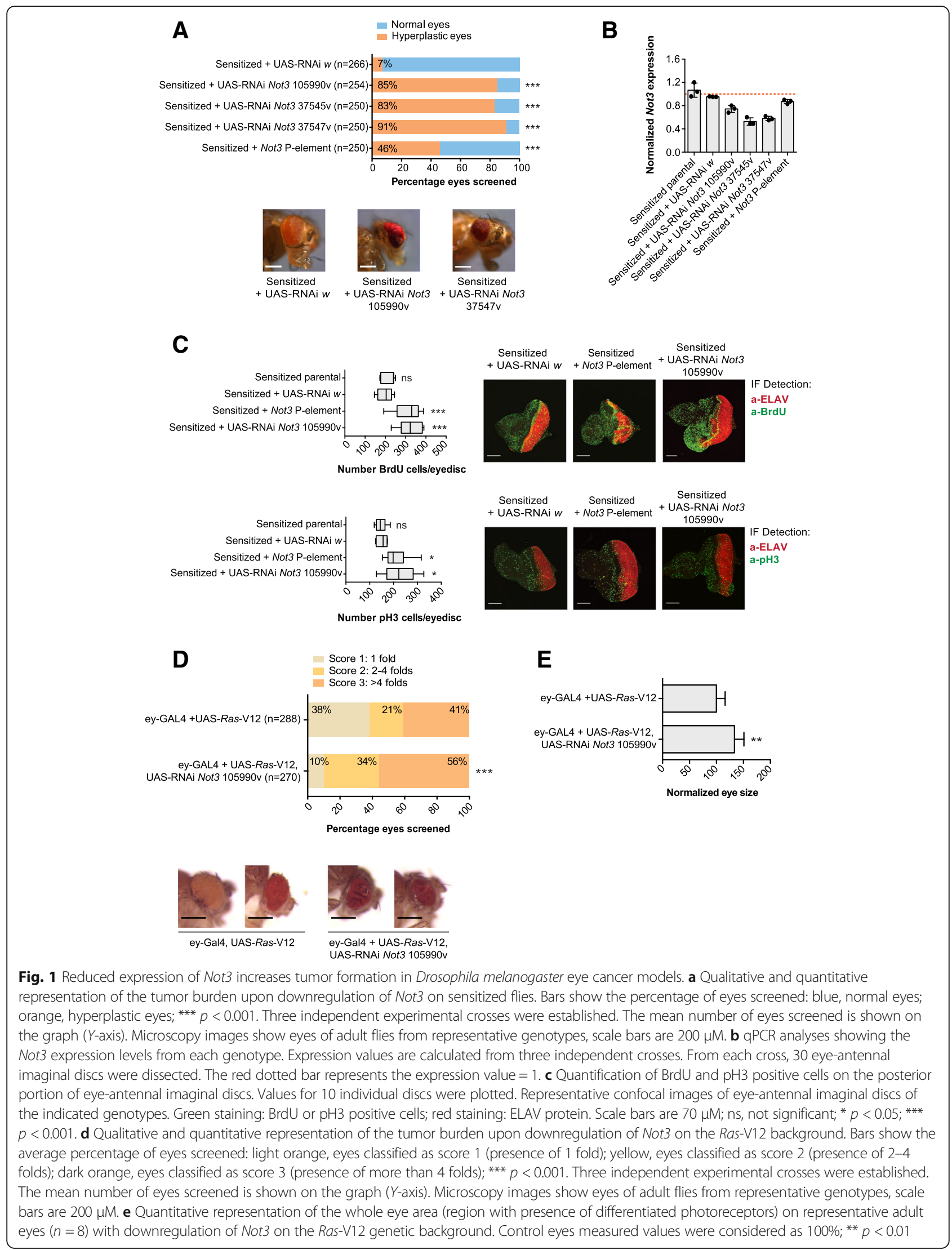


markedly disrupt the pattern of ELAV expression when compared to parental and control eye discs (Fig. 1c).

To further confirm the tumor suppressor activity of Not3 in a different context, we used a fly model that specifically overexpresses Ras-V12 in the eye. Overexpression of only Ras-V12 resulted in increased eye size and also in hyperplastic tissue with a penetrance of $62 \%$. Reduction of Not3 in this oncogenic background resulted in a marked increase in tumor incidence (90\%) and more aggressive phenotype as indicated by increased folds observed on the eye (Fig. 1d, e). Thus, reduction of Not3 expression increases proliferation of retinal precursors in the $D l$-sensitized and the Ras-V12 backgrounds.

\section{Downregulation of Not3 abrogates normal retinal differentiation}

We next aimed to dissect the physiological role of Not3 in eye development. We observed that reduction of Not3 expression in wild-type eyes (driven by ey-Gal4) resulted in a significant decrease of eye size (small rough phenotype) with a penetrance of $100 \%$ in adult flies (Fig. 2a). Expression of a UAS-RNAi white construct (control) did not change the external morphology of the eye. The Not3 P-element mutant line showed no obvious defects in retinal differentiation, maybe because the reduction of Not3 expression levels was not enough to cause defects on a wild-type tissue (Fig. 2b). These data indicate that Not3 expression is essential for proper retinal development.

We also performed immunofluorescence stainings on larval eye discs to investigate the number of $\mathrm{pH} 3 / \mathrm{BrdU}$, cleaved-DCP1a (marker of apoptosis), and ELAV positive cells. Although not significant, we observed that reduction of Not3 resulted in less cell proliferation, measured by $\mathrm{pH} 3$ and BrdU. Our results also showed that loss of Not3 led to induction of apoptosis. Differentiation was strongly inhibited, although some photoreceptors could still form in Not3-downregulated eye discs (Fig. 2c-e).

Next, we tested if downregulation of other subunits of the CCR4-NOT complex could cause similar effects. Reduction of twin (deadenylase, CNOT6/CNOT6L orthologue) or Pop2 (deadenylase, CNOT7/CNOT8 orthologue) expression led to a phenotype similar to the one observed among Not3-defective eye discs (rough small eyes) (Fig. 2d-e, Additional file 2: Figure S1A). Downregulation of Not1 (scaffold protein of the complex, CNOT1 orthologue) and Not2 (CNOT2 orthologue) was not significant, and as a consequence, the effects of Not1 or Not2 downregulation could not be assessed (Additional file 3: Figure S2A).

These results show that reduction of Not3 or the CCR4-NOT complex subunits beyond a certain threshold disrupts normal retinal differentiation and causes subsequent loss of the differentiated tissue in a wild-type background.

\section{Overexpression of Not3 suppresses tumor formation}

To investigate whether Not3 overexpression could suppress tumor formation, we first generated transgenic flies carrying a UAS-Not3 construct. Ectopic expression of Not3 was able to rescue the small rough eye phenotype previously observed in wild-type Not3-downregulated eyes, indicating that both UAS-Not3 overexpression and the effects observed upon Not3 downregulation are specific (Fig. 3a).

For subsequent experiments, we used the so-called "eyeful" flies. These flies have overexpression of the Notch ligand $D l$, together with overexpression of the epigenetic regulators lola and $p s q$. Eyeful flies display excessively enlarged eyes and eye tumors and macroscopically visible metastases derived from the developing retina [18, 25]. Overexpression of Not3 in this model significantly suppressed formation of eye tumors: $43 \%$ vs. $21 \%(p<0.01)$ when compared with UAS-GFP or $43 \%$ vs. $14 \%(p<0.001)$ when compared with UAS-LacZ. When hyperplastic eyes were detected, the tumorigenic phenotypes observed were also milder than the ones observed in the control crosses (Fig. 3b).

In the eyeful eye discs, disorganization of the epithelium as well as defects in the pattern of differentiated cells were evident (Fig. 3c). Eyeful L3 larval eye discs with Not3 overexpression had significantly less proliferating cells (less pH3/ BrdU positive cells) than control eye discs (Fig. 3c). Ectopic expression of Not3 did not significantly affect the number of apoptotic cells (cleaved-DCP1a), although it seemed there were fewer apoptotic cells when compared to the control conditions. In addition, upregulation of Not3 expression resulted in the re-establishment of the typical epithelial organization, accordingly to ELAV stainings.

Taken together, our data show that Not3 acts as a classical tumor suppressor in Drosophila melanogaster eye cancer models, with its downregulation enhancing tumor formation and its overexpression suppressing tumor formation/progression.

\section{The tumor suppressor function of Not3 is related to its function within the CCR4-NOT complex}

Since Not3 is a subunit of the CCR4-NOT complex, we asked whether downregulation of other subunits in the sensitized background causes similar effects. Reduction of expression of Not1, Not2, twin, or Pop 2 all caused increased tumor formation (Fig. 4a, b, Additional file 2: Figure S1B, and Additional file 3: Figure S2B). Of interest, reduction of twin also caused metastatic tumors (Fig. 4a). These data confirm that not only Not3, but also the entire CCR4-NOT complex functions as a tumor suppressor.

CNOT3, the human Not3 orthologue, contains three important domains. The $\mathrm{N}$-terminal region contains two coiled-coil (CC) domains, while the C-terminal region harbors a Not-box domain (NB). ClustalW2 alignment analysis 
A

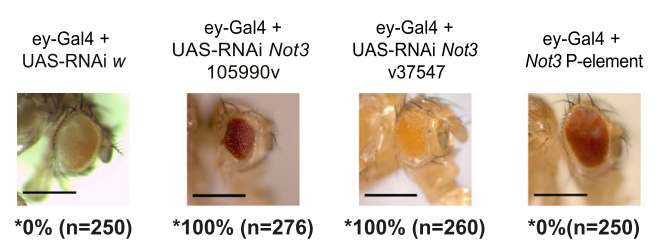

*Percentage of aberrant eyes in $\mathrm{F} 1$
B

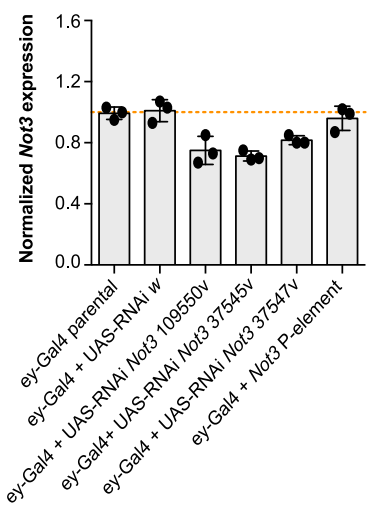

C
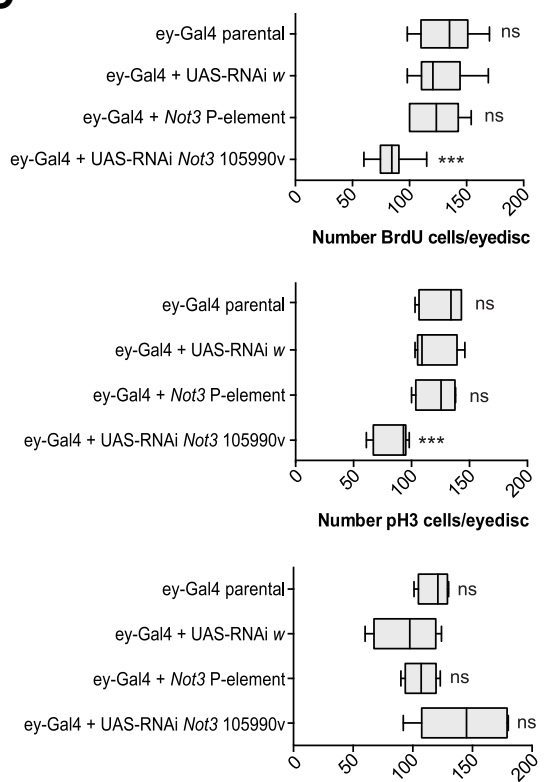

Number cleaved-DCP1a cells/eyedisc

D

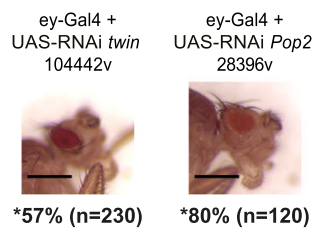

*Percentage of aberrant eyes in $\mathrm{F} 1$

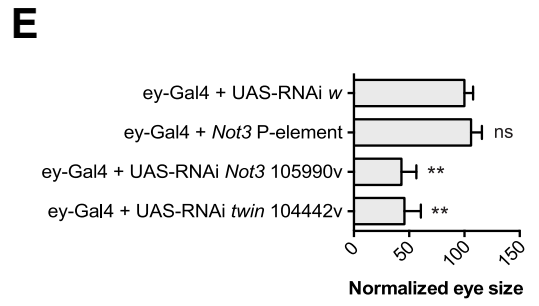

Fig. 2 Reduced expression of Not3 in a wild-type genetic background abolishes normal retinal differentiation. a Microscopy images show eyes of adult flies with Not3 reduced expression levels from representative genotypes, scale bars are $200 \mu \mathrm{M}$. The percentage of F1 "small rough" defective eyes and the mean number of eyes screened are indicated on the bottom side of each image. Three independent crosses were performed for each experimental condition. $\mathbf{b}$ qPCR analyses showing the Not3 expression levels from each genotype. Expression values are calculated from three independent crosses. From each cross, 30 eye-antennal imaginal discs were dissected. The red dotted bar represents the expression value $=1$. c Quantification of BrdU, pH3, and cleaved-DCP1a positive cells on the posterior portion of eye-antennal imaginal discs $(n=10)$. Representative confocal images of eye-antennal imaginal discs of the indicated genotypes. Green staining: BrdU, pH3, or cleaved-DCP1a positive cells; red staining: ELAV protein. Scale bars are $70 \mu \mathrm{M}$; ns, not significant; ${ }^{* * *} p<0.001$. d Microscopy images show eyes of adult flies with downregulation of the Pop2 and twin CCR4-NOT complex subunits on a wild-type background, scale bars are $200 \mu \mathrm{M}$. The percentage of F1 "small rough" defective eyes and the mean number of eyes screened are indicated on the bottom side of each image. $\mathbf{e}$ Quantitative representation of the whole eye area (region with presence of differentiated photoreceptors) on representative adult eyes $(n=8)$ with downregulation of Not3 or twin on the wild-type genetic background. Control eyes measured values were considered as 100\%; ns, not significant; ** $p<0.01$ 


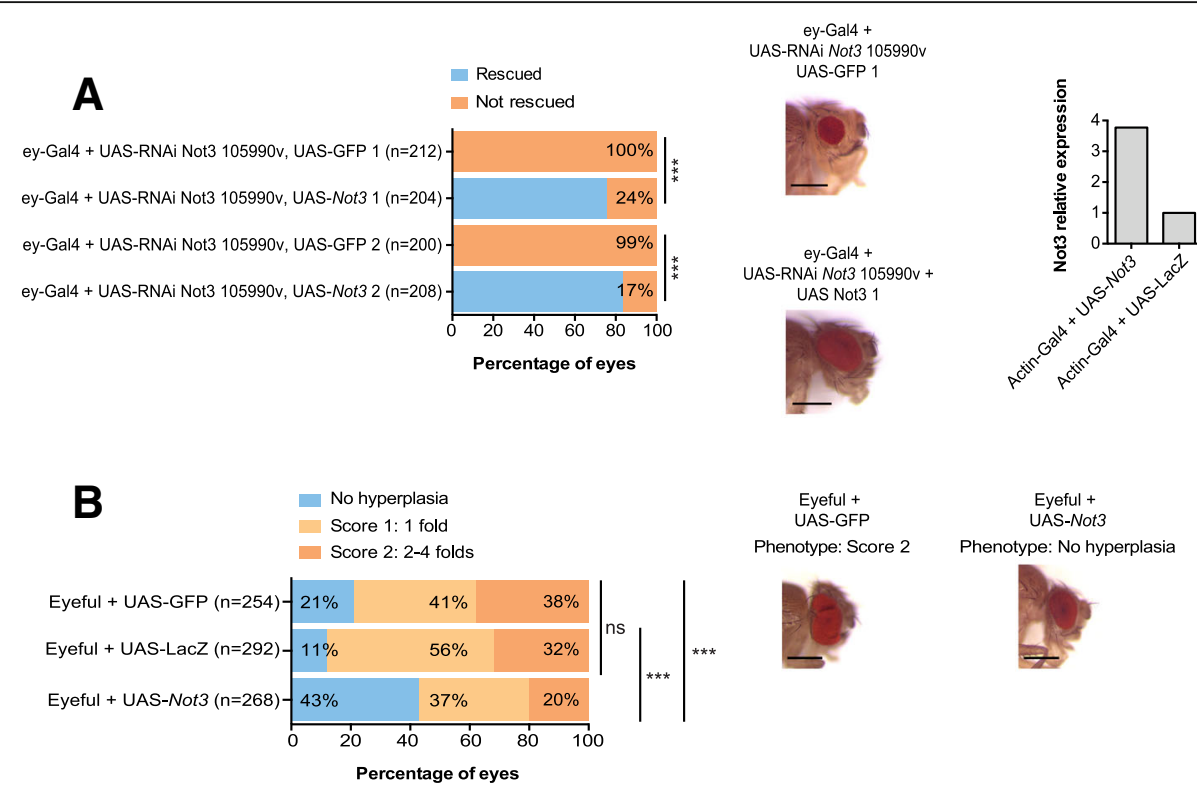

\section{C}
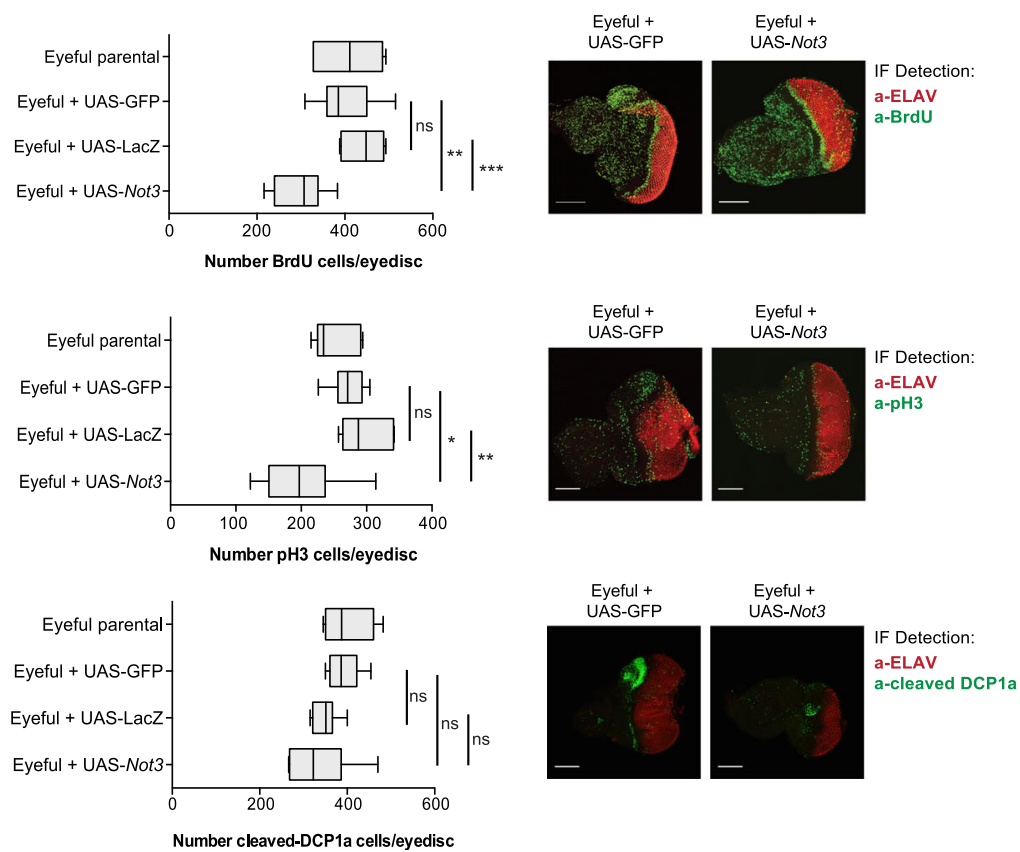

Fig. 3 Ectopic expression of Not3 suppresses tumor formation in eyeful flies. a Qualitative and quantitative representation of the defective eyes upon ectopic expression of Not3 on wild-type flies with reduced Not3 expression. Bars show the percentage of eyes screened: blue, rescued eyes; orange, not rescued eyes; ${ }^{* * *} p<0.001$. Numbers 1 and 2 on each genotype represented on the graph refer to different recombinant clones. Three independent crosses were analyzed. The mean number of eyes screened is shown on the graph ( $Y$-axis). Microscopy images show eyes of adult flies from representative genotypes, scale bars are $200 \mu \mathrm{M}$. qPCR analyses showing the Not3 expression levels from each genotype. Each bar represents the expression value of a pool of 10 individuals. $\mathbf{b}$ Qualitative and quantitative representation of the hyperplastic eyes upon ectopic expression of Not3 on eyeful flies. Bars show the percentage of eyes screened: blue, eyes with no hyperplasia; light orange, eyes classified as score 1 (presence of 1 fold); dark orange, eyes classified as score 2 (presence of $2-4$ folds); ${ }^{* *} p<0.01,{ }^{* * *} p<0.001$. Three independent crosses were analyzed. The mean number of eyes screened is shown on the graph (Y-axis). Microscopy images show eyes of adult flies from representative genotypes. Scale bars are $200 \mu$ M. c Quantification of BrdU, pH3, and cleaved-DCP1a positive cells on the posterior part of L3 larval eye-antennal imaginal discs. Values for 10 individual discs were plotted. Representative confocal images of eye-antennal imaginal discs of the indicated genotypes. Green staining: $\mathrm{BrdU}$, pH3, or cleavedDCP1a positive cells; red staining: ELAV protein. Scale bars are $70 \mu \mathrm{M}$; ns, not significant; ${ }^{*} p<0.05 ;{ }^{* *} p<0.01 ;{ }^{* *} p<0.001$ 


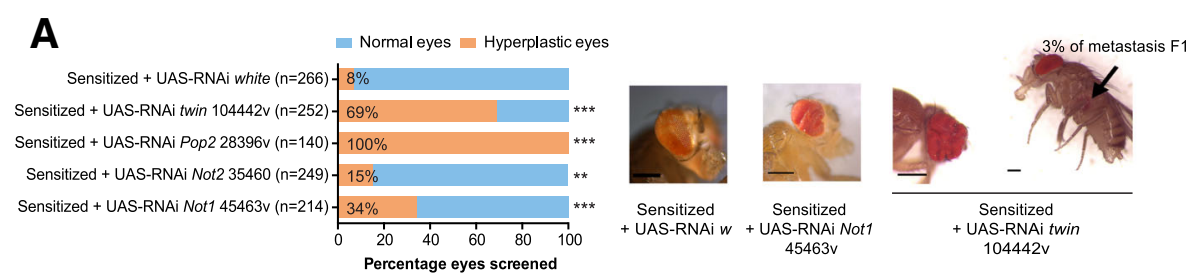

B

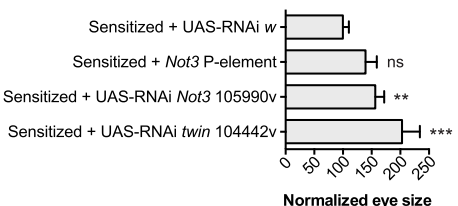

C

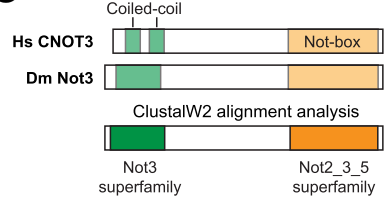

D

Rescued Almost rescued No rescued

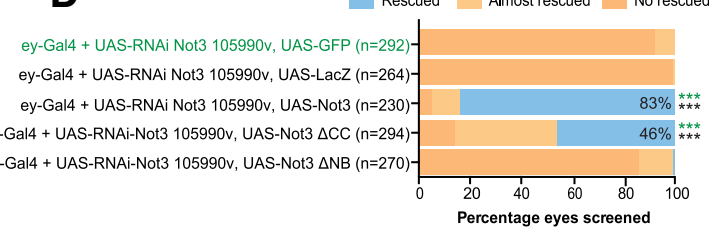

Designed UAS-constructs:
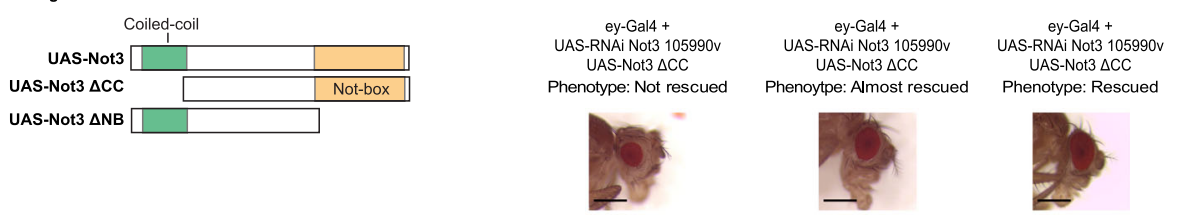

$\mathbf{E}$

$\mathbf{F}$
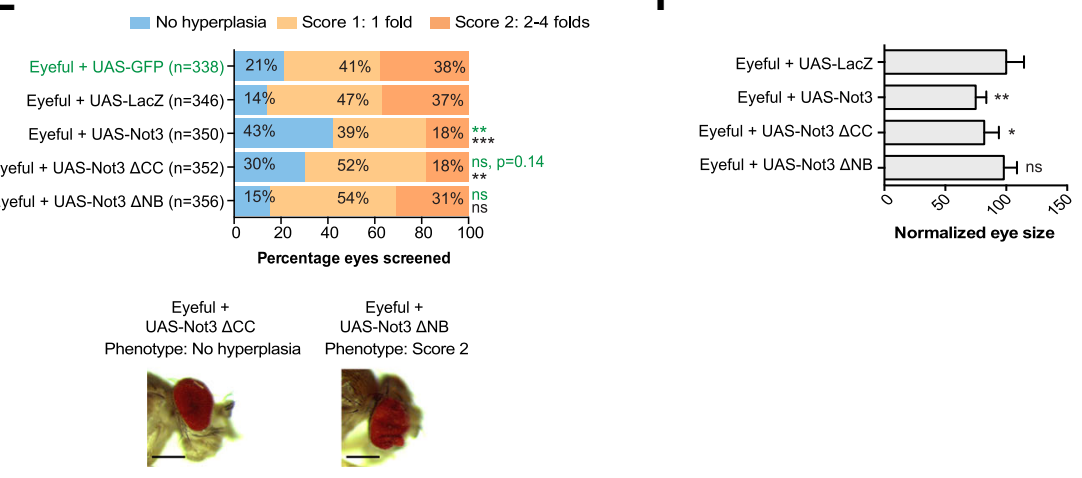

Eyeful +

Phenotype: Score 2

Fig. 4 (See legend on next page.) 


\section{(See figure on previous page.)}

Fig. 4 The Not3 Not-box domain is important for normal protein functionality. a Qualitative and quantitative representation of the tumor burden upon downregulation of different CCR4-NOT complex subunits on the sensitized background. Bars show the percentage of eyes screened: blue, normal eyes; orange, hyperplastic eyes; ${ }^{* *} p<0.01 ;{ }^{* * *} p<0.001$. The mean number of eyes screened is shown on the graph ( $Y$-axis) and three independent crosses were established. Microcopy images show the eyes of adult flies from representative genotypes. Scale bars are $200 \mu \mathrm{M}$. Black arrow indicates the presence of metastasis. b Quantitative representation of the whole eye area (region with presence of differentiated photoreceptors) on representative adult eyes $(n=8)$ with downregulation of Not3 or twin on the sensitized genetic background. Control eyes measured values were considered as 100\%; ns, not significant; ${ }^{* *} p<0.01 ;{ }^{* * *} p<0.001$. c Schematic representation of the human CNOT3 and the fly Not3 proteins. ClustalW2 alignment analyses show that the coiled-coil (green) and Not-box (orange) regions are present in both species. Designed UAS constructs are also shown: UAS-Not3 (wild-type Not3), UAS-Not3 $\triangle C C$ (deletion of the coiled-coil domain region), UAS-Not3 $\triangle N B$ (deletion of the Not-box domain). $\mathbf{d}$ Qualitative and quantitative representation of the defective eyes upon ectopic expression of Not3, Not3- $\Delta C C$, and Not3- $\Delta N B$ on wild-type flies with reduced Not3 expression. The mean number of eyes screened is shown on the graph (Y-axis), and three independent crosses were established. Bars show the percentage of eyes screened: blue, normal eyes; light orange, eyes classified as "almost rescued"; dark orange, eyes classified as "not rescued"; *** $p<0.001$. In green color, comparisons to UAS-GFP control flies; black color, comparison to UAS-LacZ control flies. Microscopy images show the eyes of adult flies from each category. Scale bars are $200 \mu \mathrm{M}$. e Qualitative and quantitative representation of the defective eyes upon ectopic expression of Not3, Not3- $\Delta C C$, and Not3- $\Delta$ NB on eyeful flies. Bars show the percentage of eyes screened: blue, normal eyes; light orange, eyes classified as score 1 (presence of 1 fold); dark orange, eyes classified as score 2 (presence of 2-4 folds); ns, means not significant, ** $p>0.01,{ }^{* * *} p<0.001$. The mean number of eyes screened is shown on the graph ( $Y$-axis). In green color, comparisons to UAS-GFP control flies; black color, comparison to UAS-LacZ control flies. Microscopy images show the eyes of adult flies from each category. Scale bars are $200 \mu \mathrm{M}$. f Quantitative representation of the whole eye area (region with presence of differentiated photoreceptors) on representative adult eyes $(n=8)$ with ectopic expression of Not3 wild-type or Not3 mutants on the eyeful genetic background. Control eyes measured values were considered as 100\%; ns, not significant; ${ }^{*} p<0.05$; ${ }^{* *} p<0.01$

showed that the CC and NB domains are conserved in the Not3 protein from Drosophila melanogaster (Fig. 4c). We then characterized which Not3 domains are essential for tumor suppression in the eyeful background. We designed and cloned two versions of the wild-type Not3 open reading frame in a pUASTattB vector: UAS-Not3- $\triangle C C$ (lacking the CC domain) and UAS-Not3- $\triangle \mathrm{NB}$ (lacking the NB domain) (Fig. 4c). First, we measured whether the expression levels achieved by ectopic expression of these constructs were similar. Since a good antibody against the Not3 protein from Drosophila melanogaster was not available, we co-transfected S2 cells with a pMT-Gal4 plasmid and a flag-tagged version of each of the UAS-Not3 plasmids. Cycloheximide treatment of the transfected cells was also performed in order to investigate the stability of the translated proteins. Our results showed that similar expression levels were achieved with all plasmids and that the translated proteins were equally stable (Additional file 4: Figure S3A).

Next, we engineered transgenic flies expressing the different versions of the wild-type Not3 open reading frame (without a flag-tag). Since Not3 is on chromosome 2 on flies, we decided to insert our UAS-Not3 transgenes on chromosome 3 (site 89E11). Our qPCR analyses showed that ectopic expression of UAS-Not3 plasmids driven by Actin5C-Gal4 results in similar expression levels among all constructs used in these experiments (Additional file 4: Figure S3B).

Ectopic expression of UAS-Not3- $\Delta$ CC in Not3-defective flies rescued the Not3 small rough eye phenotype previously observed, while no rescue was observed upon expression of UAS-Not3- $\triangle \mathrm{NB}$ (Fig. 4d). Similar results were observed in the eyeful background. Overexpression of UAS-Not3- $\Delta C C$ had a mild effect tumor formation with $30 \%$ penetrance, while UAS-Not3- $\Delta$ NB did not suppress the tumor phenotype at all (Fig. 4e, f).

Taken together, our results show that the NB domain is essential to rescue developmental and cancer phenotypes observed upon downregulation of Not3. Since the NB domain is essential for the association of Not3 with the CCR4-NOT complex $[27,28]$, these data further indicate that the effects observed with downregulation of Not 3 are linked to its function within the CCR4-NOT complex.

\section{Not3 disruption in the sensitized background causes an aberrant gene expression program}

The major role of the CCR4-NOT complex is to regulate mRNA stability through deadenylation of mRNA. To determine which mRNA transcripts are affected by Not3 knockdown, we carried out RNA-sequencing analysis (Fig. 5a) on L3 larvae eye discs isolated from the sensitized parental, sensitized control (sensitized + UAS-RNAi white), and sensitized Not3-tumor eye discs: sensitized + Not3-Pelement and sensitized + UAS-RNAi Not3 knockdowns. Correlations were calculated between the mRNA expression profiles of the different samples. High correlations were observed between the different Not3 knockdown samples. The sensitized + Not3 P-element samples were more closely related to the control samples, which is in agreement with mild reduction of Not3 in this model and mild phenotypic effects observed (Fig. 5b).

A differential gene expression analysis was performed between the different Not3 knockdown models and the sensitized parental, confirming the significant downregulation of Not3 mRNA levels with an average log2 fold change of -0.2270 . Moreover, these analyses revealed that 
A

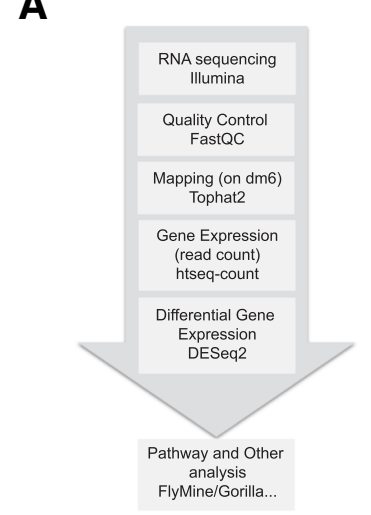

C

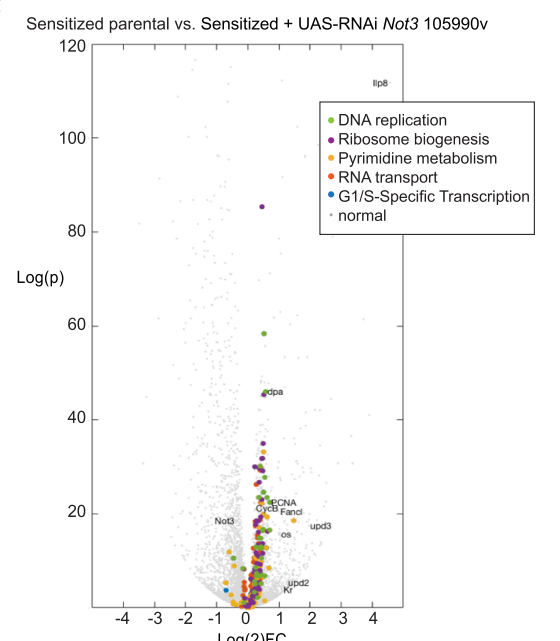

E

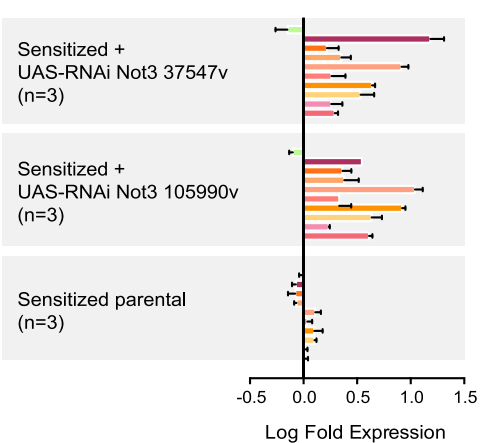

B

$\mathbf{F}$
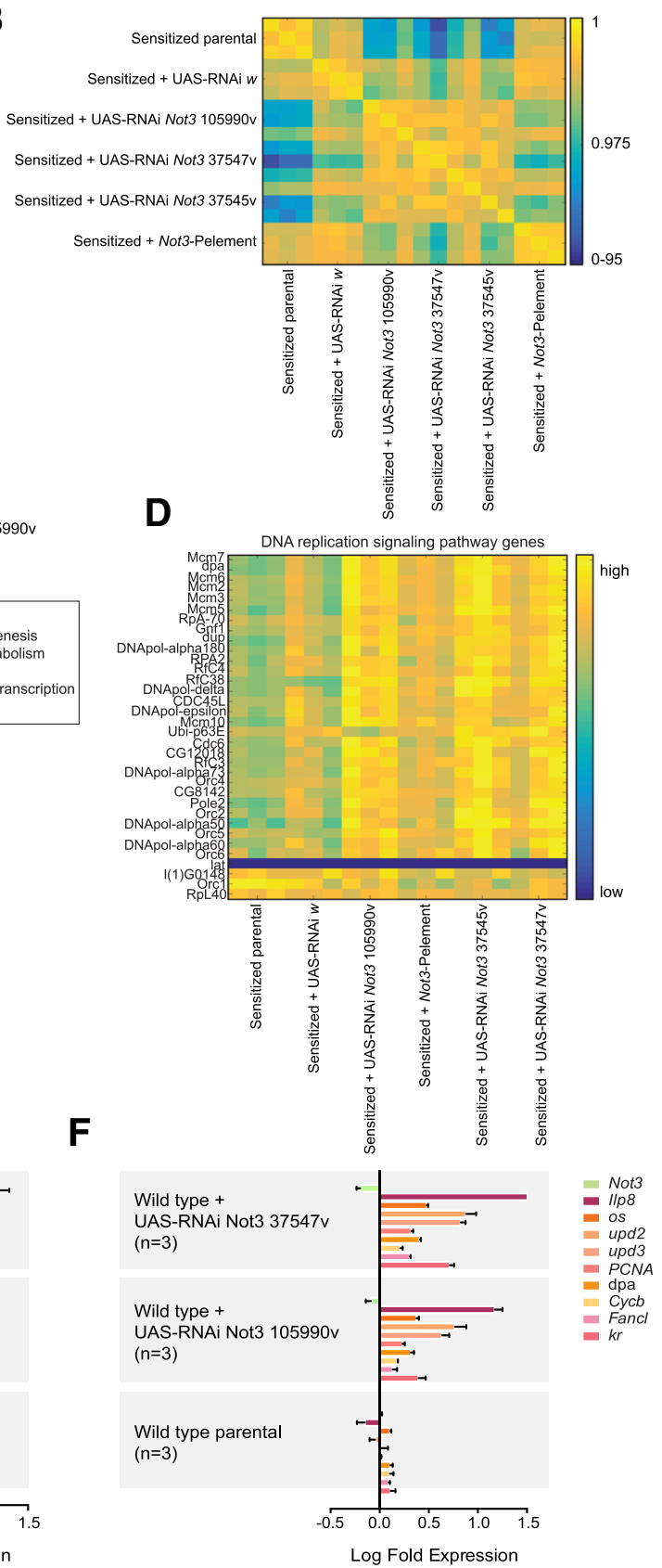

Fig. 5 Not3 reduced expression in the sensitized background drives oncogenic altered gene expression. a Workflow for the analysis of the RNA-sequencing experiments performed on fly eye-antennal eye discs. $\mathbf{b}$ Correlation matrix between the different samples, calculated on the normalized mRNA expression levels. c Volcano plot of all differential genes between sensitized + UAS-RNAi Not3 105990v and sensitized parental, which represents the results of three biological replicates. The other Not3 knockdowns show similar results. The genes belonging to one of the most enriched signaling pathways are marked in color. $\mathbf{d}$ Matrix showing the normalized mRNA expression levels of those genes that belong to the DNA replication signaling pathway. e qPCR validation experiments of selected genes on the sensitized + Not3 defective tumor models. Data were normalized accordingly to the expression levels on the sensitized + UAS-RNAi white eye-antennal discs. Results are plotted as mean \pm standard deviation. $\mathbf{f}$ qPCR validation experiments of selected genes on the ey-Gal4 Not3 defective models. Data were normalized accordingly to the expression levels on the ey-Gal4 + UAS-RNAi white eye-antennal discs. Results are plotted as mean \pm standard deviation

approximately 3000 significant differential genes were found on each of the Not3 knockdowns performed (Additional file 1: Tables S3-S6). Combining the different gene lists revealed 1028 misregulated genes, with 448 and 580 significantly up- and downregulated, respectively (Additional file 1: Table S7). 
Gene set enrichment analysis (GSEA) identified DNA replication and ribosome biogenesis as significantly enriched signaling pathways $(p=4.81 \mathrm{E}-6$ and $p=$ $9.68 \mathrm{E}-4$, respectively) (Fig. 5c). Notably, we found that 30 out of 34 genes from the DNA replication pathway were significantly upregulated (Fig. 5d). Metabolic, RNA transport and cell cycle signaling pathways were also highly enriched (Additional file 1: Table S8). Of importance, we only identified significantly enriched signaling pathways among the upregulated genes (Fig. 5e). These findings strongly support that the upregulated genes are specific effects of Not3 knockdown, while downregulated genes may represent secondary effects.

Besides, we also detected upregulation of genes that could be linked to tumorigenesis. These included the pupation regulator insulin-like peptide 8 (Ilp8), the three unpaired $(u p d)$ genes (which encode the ligands for the JAK/STAT pathway), the cyclin B (Cycb) cell cycle regulator, and the discs proliferation abnormal (dpa) and the PCNA genes (Additional file 1: Table S7). Increased expression of those selected genes was further validated by using qPCR on the sensitized and the wild-type models. These results show that downregulation of Not3 causes the upregulation of those genes specifically and independently of $D l$ ectopic expression (Fig. 5f).

In order to clarify whether Not3 regulates mRNA decay of those target genes or controls the transcription of those genes, we performed an i-CisTarget analysis on the upregulated genes to determine if Not3 DNA-binding sites were present in the promoters of the upregulated genes. We did not see any enrichment of the described Not3 motif [29] (NES = 0.72). Moreover, with PWMtools, we looked for occurrences of the Not3 motif in the promoter sites of these upregulated genes and we found that only a small portion of the genes, not more than expected by chance, has Not3 binding motifs. Next, we tested the stability of some target genes in vitro using Drosophila S2 cells (Additional file 5: Figure S4A-C). Our results show a clear knockdown of the Not3 mRNA expression (almost $50 \%$ efficiency) at time point $0 \mathrm{~h}$, which was associated with significant upregulation of $C y c b, f a n c l$, and $u p d 2$ (Additional file 5: Figure S4B). No significant changes were detected on the expression level of CG1239, a gene we used as a control, since its expression was not significantly changed on our RNA-sequencing experiments after Not3 downregulation (Additional file 5: Figure S4B). Analysis after transcription block provided a view on mRNA stability and changes induced by Not3 downregulation. $C y c b$, fancl, and upd2 mRNAs showed a moderate increase in stability after Not3 downregulation when compared with the control condition (YFP expression). Again, no effects were observed on the mRNA stabilization of CG1239 (Additional file 5: Figure S4C), indicating that the effects observed are specific. In conclusion, our results show that Not3 downregulation causes an upregulation of $C y c b, f a n c l$, and upd 2 transcripts in S2 cells and that this can be linked to increased mRNA stability.

\section{Upregulation of CycB contributes to the Not3 tumor phenotype}

We decided to investigate the function of seven selected target genes (Cycb, os, upd2, upd3, Fancl, PCNA, and $d p a)$ on tumor development by knocking down their expression in the sensitized + Not3-tumor model (independent RNAi constructs/loss-of-function mutants were tested per gene). Before doing that, we excluded the possibility that downregulation of the selected genes could already cause defects on the wild-type retina. Downregulation of PCNA and $d p a$ led to significant developmental defects on the fly's retina, preventing their study in the eye tumor models.

For the other genes, we quantified effects on eye tumor rescue. Knockdown of $C y c B$ on the sensitized + Not3-tumor model yielded the strongest effects. The $c y c B$ [2] mutant rescued the tumor phenotype to over $50 \%$ compared to the control RNAi construct ( 15\% for white RNAi). Knockdown of fancl and JAK-STAT ligands had minor effects. For instance, knockdown of upd2/ upd 3 showed rescue of tumor formation in $28 \%$ of eyes screened. A trend towards significance $(p=0.06)$ was observed on tumor rescue upon knockdown of upd3 only (Fig. 6a). Genetic suppression of os and upd3 on the sensitized + Not3-tumor animals caused pupal lethality, precluding its analysis. If tumor development associated with Not3 disruption is linked to the CCR4-NOT complex functionality, downregulation of selected genes could also rescue tumor development in the sensitized + twin-tumors. Again, genetic suppression of $C y c b$ yielded the strongest effects, with the $c y c b$ [2] mutant rescuing tumor phenotype to over 50\% compared to the control RNAi construct $(\sim 10 \%$ for white RNAi) (Fig. 6a). Knockdown of fancl and genetic suppression of the JAK-STAT ligands on the sensitized + twin background showed a significant effect on tumor rescue (Fig. 6a).

Examination of expression of a 10xSTAT92E-GFP reporter for JAK-STAT pathway activation [30] by immunofluorescence upon Not3 or twin downregulation on the sensitized model was then performed. We observed a significant increase of the 10xSTAT92E-GFP expression domains (Fig. 6b-d). However, since knockdown of JAK-STAT ligands had minor effects on tumor growth rescue, especially upon Not3 downregulation, it might be that the pathway is active due to alternative mechanisms.

Taken together, our results show that upregulation of $C y c B$ contributes to the tumor phenotype in our sensitized Not3 and twin tumor models. 

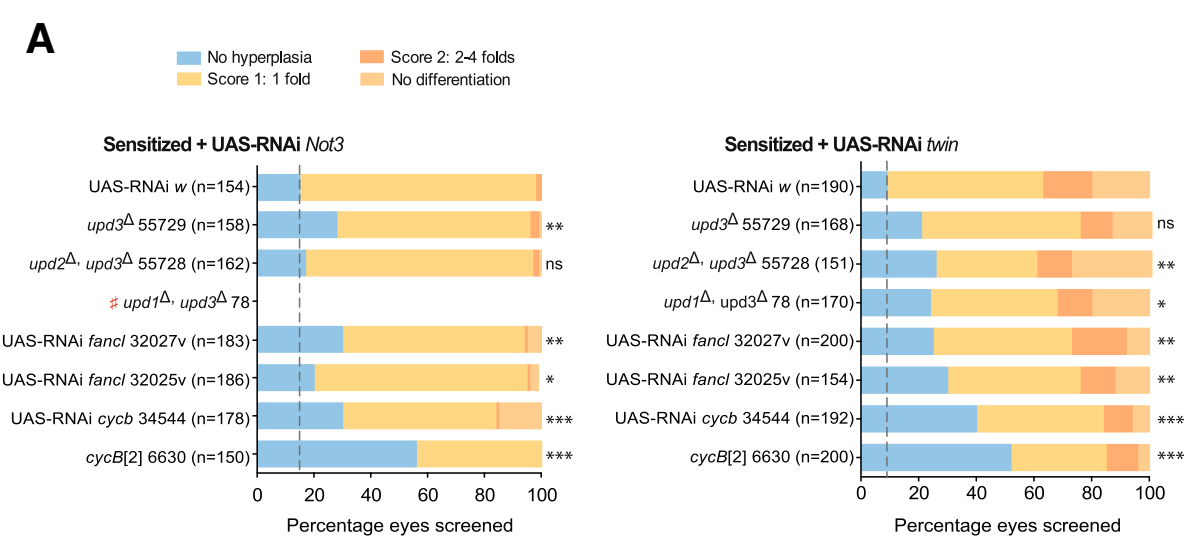

\#Genetic suppression of os and upd 3 caused pupal lethality

B

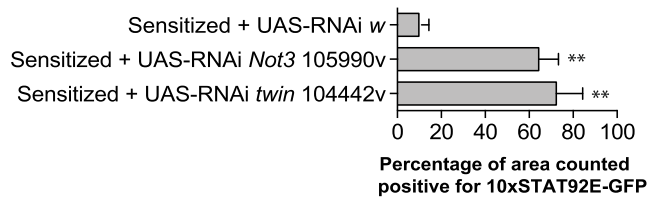

C

positive for 10xSTATg2E-GFP

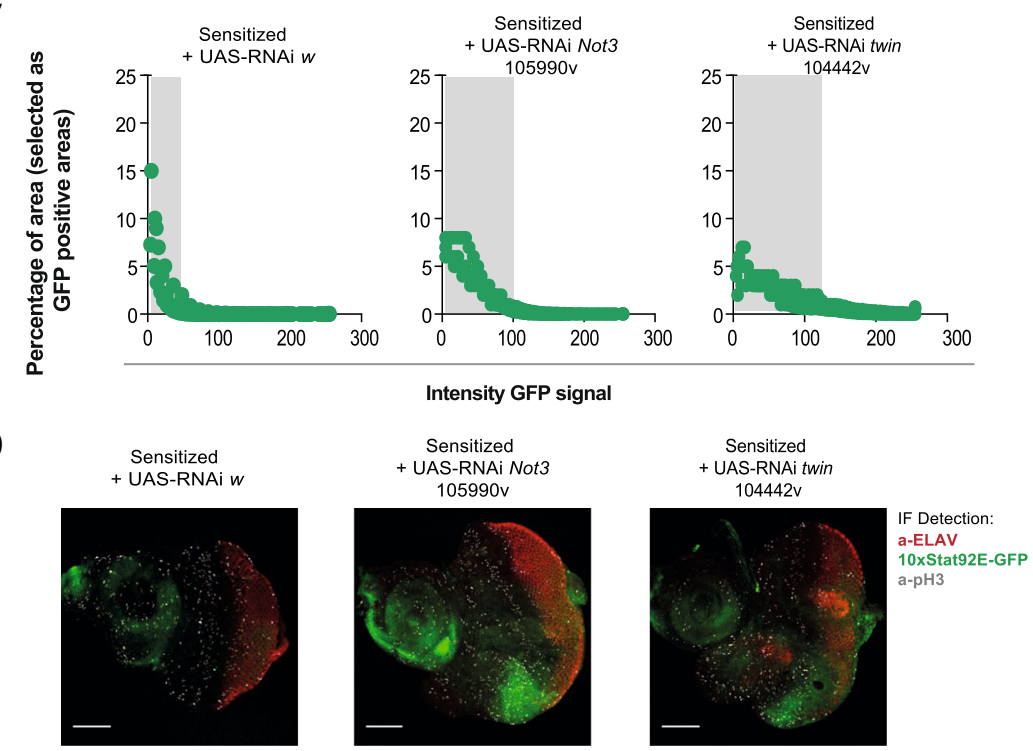

Fig. 6 Upregulation of $c y c B$ contributes to the tumor phenotype in Not3 and twin sensitized tumor models. a Sensitized + Not3 defective tumor model and sensitized + twin defective tumor model. Bar graphs showing rescue scoring results for tumor model or tumor plus knockdown for the indicated gene. Two independent RNAis/mutant lines are shown. Three independent crosses were screened on each experimental condition. The mean number of eyes analyzed is shown on the graph ( $Y$-axis). Genetic suppression of os and upd3 on the sensitized + Not3 tumor animals caused pupal lethality precluding its analysis. Blue, no hyperplasia; yellow, score 1: 1 fold; light orange, score 2: 2-4 folds; dark orange, no differentiation; gray, no data available. ns, not significant; * $p<0.05$; ${ }^{* *} p<0.01 ;{ }^{* *} p<0.001$. b Quantification of 10xSTAT92E-GFP expression domains on the posterior part of $L 3$ wandering larval eye-antennal discs using the color inspector 3D ImageJ plugin. GFP expression domains were measured by analysis of confocal micrographs and expressed as a percentage of the posterior eye-disc area. Bar graphs show the percentage of areas positive for GFP signal (thus, areas with JAK-STAT signaling activation) from the different genotypes; ${ }^{* *} p<0.01$. Six eye-antennal discs were analyzed per experimental condition. Experimental crosses were performed in triplicate. Graph shows results from one representative experiment. c Dot graphs show the green signal intensity of those areas previously selected as GFP positive areas. We observed that both the area of GFP expression domains and the green intensity of those areas are increased in sensitized $+\operatorname{Not3}(n=6)$ or sensitized + twin tumor eye-antennal discs $(n=6)$. Experimental crosses were performed in triplicate; graph shows results from one representative experiment. $\mathbf{d}$ Representative confocal images of eye-antennal imaginal discs of the indicated genotypes. Green signal: 10xSTAT92E-GFP; red signal: ELAV protein; white signal: pH3 positive cells. Scale bars are $50 \mu \mathrm{M}$ 
A

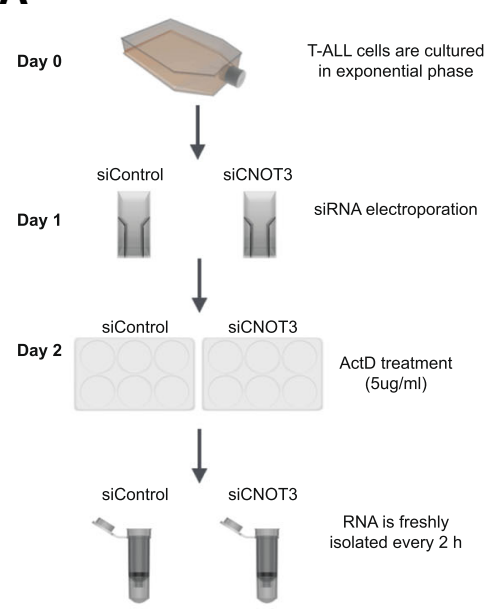

B

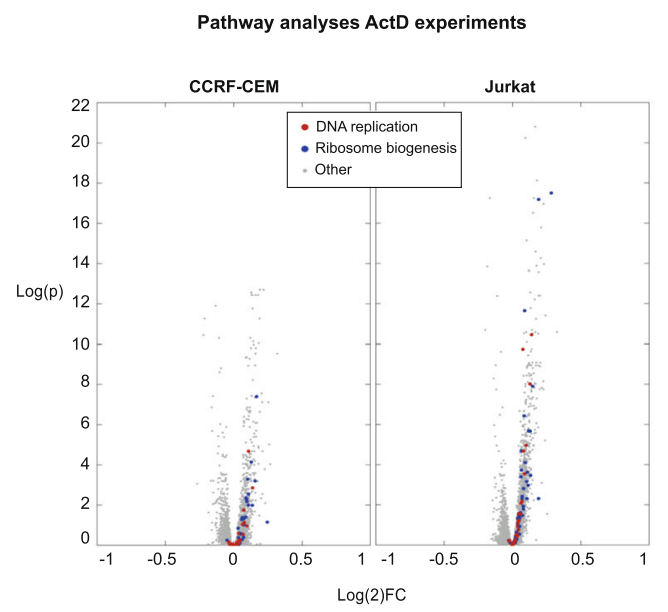

C CCRF-CEM
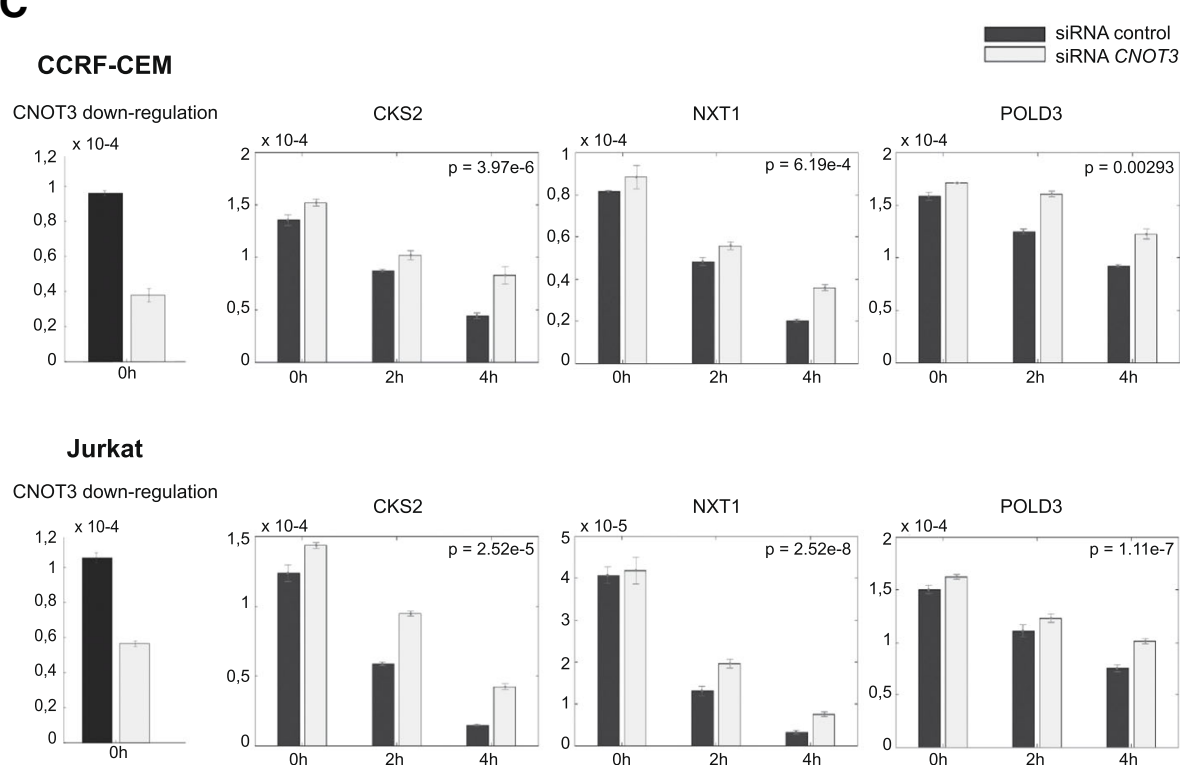

Fig. 7 Loss of CNOT3 leads to stabilization of DNA replication and ribosome biogenesis signaling components in human T-ALL cell lines. a Workflow for the Actinomycin D experiments upon CNOT3 downregulation on T-ALL cell lines. Experiments were performed in three independent series. $\mathbf{b}$ Volcano plots from Jurkat and CCRF-CEM T-ALL cell lines. Those genes belonging to one of the most enriched signaling pathways are marked in color. $\mathbf{c}$ mRNA expression levels of CNOT3 in all conditions and mRNA expression levels of three exemplary genes that become more stable upon CNOT3 knockdown, i.e., they show an increasing difference between the knockdown and the wild-type. Experiments were performed in three independent series

Loss of CNOT3 leads to stabilization of DNA replication and ribosome biogenesis signaling components in human T-ALL cell lines

Thereafter, we tested if the results obtained in Drosophila could be translated to human T-ALL, since CNOT3 mutations were described in this tumor type. We knocked down CNOT3 in human T-ALL cell lines JURKAT and CCRF-CEM by using siRNAs (two CNOT3 specific and a negative control siRNA). We determined effects on steady-state mRNA expression levels and also assessed the effect on RNA stability by blocking transcription and measuring the half-life of mRNA's in a global way by
RNA-seq. We stopped the transcription of newly synthesized mRNA by treating cells with Actinomycin D $24 \mathrm{~h}$ after electroporation. RNA was isolated at $0 \mathrm{~h}$ (reference time point), $2 \mathrm{~h}$, and $4 \mathrm{~h}$ time points after Actinomycin $\mathrm{D}$ treatment and submitted to RNA-sequencing (Fig. 7a).

As expected, we observed a clear knockdown of the CNOT3 mRNA expression (almost 50\% efficiency) at time point $0 \mathrm{~h}$ (Fig. 7b), which was associated with the upregulation of 975 and 1611 transcripts in JURKAT and CCRF-CEM cells, respectively (Additional file 1: Tables S9 and S10). Pathway analysis with DAVID revealed that, similar to our findings in Drosophila, the DNA replication pathway 
and the ribosome biogenesis pathways were significantly enriched (Fig. 7c, Additional file 1: Tables S11-S13) in both cell lines. RNA-seq analysis at $2 \mathrm{~h}$ and $4 \mathrm{~h}$ after transcription block provided a genome-wide view on mRNA stability and changes induced by CNOT3 downregulation. Among the significantly upregulated and more stable genes were genes involved in DNA replication pathway and the ribosome biogenesis including NXT1, POLD3, and CKS2 (Fig. 7c, Additional file 1: Tables S12 and S13).

\section{Discussion}

Mutations on CNOT3, and more rarely in other members of the CCR4-NOT complex, have recently been identified in T-ALL and chronic lymphoblastic leukemia $[13,31]$. In solid tumors, recent reports show that CNOT3 and other CCR4-NOT subunits could be involved in tumor formation/progression. Downregulation of Cnot2 expression results in a significant increase of breast cancer pulmonary metastasis in vivo [32]. Instead, CNOT3 confers an aggressive behavior in colorectal cancer cells through a self-renewal transcriptional program [33]. Mutations on CNOT3 have been found in T-ALL patients experiencing treatment failure after first relapse, although this observation needs to be confirmed in a larger cohort of patients [34]. Thus, it seems that depending on the cellular context, those proteins can have either tumor suppressor or oncogenic properties. However, it still remains unclear how mutations on CNOT3 or other CCR4-NOT subunits can contribute to tumor development.

In this report, we show that Not 3 behaves as a classical tumor suppressor, with reduction of Not3 expression leading to increased tumor formation and overexpression of Not3 leading to suppressed tumor formation. Interestingly, and in agreement with sequencing data in human cancers, we also observed increased tumor formation upon knockdown of other members of the CCR4-NOT complex, including the deadenylases. These data make clear that the entire CCR4-NOT complex functions as a tumor suppressor complex and that this is likely, at least in part, through its function in mRNA metabolism.

In human cancer, only one allele of CNOT3 is mutated, suggesting that this is sufficient to contribute to tumor development and that complete loss of CNOT3 is not viable for the cells. In fact, our results show that little reduction on Not3 expression levels in a sensitized/ pre-neoplastic background is sufficient to initiate and dramatically increase tumor formation. Conversely, a little reduction on Not3 expression is not enough to cause defects on wild-type tissues. This is consistent with previous data showing that the presence of only one Cnot3 allele is enough to sustain normal cell development [6].

Recent structure-function analyses have revealed that CNOT2 and CNOT3 bind to the CCR4-NOT complex through the Not-box domain [27, 28]. We demonstrate that overexpression of a Not3 mutant lacking the Not-box domain does not rescue neither the Not3-downregulated nor the eyeful tumor phenotype. These data confirm that the defects observed in our models are related to the function of Not3 within the CCR4-NOT complex. In agreement, it has been shown that expression of a CNOT3 mutant lacking the Not-box domain in CNOT3-depleted mouse embryonic fibroblasts was not able to rescue proper formation of the CCR4-NOT complex and the decreased cell viability [35].

To further determine the exact consequences of downregulation of Not3, we performed RNA-sequencing on Drosophila eye discs. CNOT3 was described to be involved in transcriptional regulation [26, 32, 35]. However, we did not find evidence that Not3 is directly involved in the transcription of the genes that were upregulated upon Not3 knockdown, as the Not3 DNA-binding motif was not enriched in the promoters of that gene set. Our findings revealed an important role for Cyclin $\mathrm{B}(\mathrm{CycB})$, a gene known to be upregulated in various cancers [36, 37]. We observed that $C y c B$ was upregulated upon Not3 downregulation and stabilized upon Not3 downregulation in S2 cells in vitro. Moreover, downregulation of $C y c B$ counteracted tumor formation, illustrating an important role for $C y c B$ in these tumors.

Our RNA-sequencing analyses also revealed that genes involved in DNA replication and ribosome biogenesis are significantly increased upon Not3 downregulation. Moreover, we confirmed on human cell lines that the increased mRNA expression level of those genes is due to mRNA stabilization, and not to a significant increased on gene transcription rates. Increased expression of genes involved in DNA replication has been found in cancer cells [38] and might result in increased replication initiation activity at a global level. There is growing evidence that an upregulated ribosome biogenesis might provide an increased risk of cancer onset [39]. Aberrant ribosome synthesis contributes to increased cellular proliferation [40, 41]. Differential expression of several ribosomal protein genes has been observed in cancer $[39,42]$. Thus, Not3/CNOT3 loss could contribute to cancer development through those two signaling pathways.

\section{Conclusions}

- We establish for the first time that Not3 and other members of the CCR4-NOT complex act as tumor suppressor genes.

- Our results indicate that the entire CCR4-NOT complex serves as a tumor suppressor, in part by suppressing transcripts implicated in DNA replication and RNA biogenesis. 


\section{Additional files}

Additional file 1: Table S1. Fly stocks used in the present study. Table S2 Primers used for cloning and real-time PCR. Table S3 Differential gene analysis. Comparison using the sensitized + UAS-RNAi Not3 105990v as target. Table S4 Differential gene analysis. Comparison using the sensitized + UAS-RNAi Not3 37547v as target. Table S5 Differential gene analysis. Comparison using the sensitized + UAS-RNAi Not3 37545V as target. Table S6 Differential gene analysis. Comparison using the sensitized + Not3 P-element as target. Table S7 List of core differential genes in all 4 comparisons. Table S8 Pathway analyses derived from the gene eye tumor signature, results from a FlyMine analysis. Table S9. Differential genes in Actinomycin D experiments on JURKAT cells. Table S10 Differential genes in Actinomycin D experiments on CCRF-CEM cells. Table S11 Description of the KEGG gene sets. Table S12 KEGG analyses on JURKAT cells. Table S13 KEGG analyses on CCRF-CEM cells. (XLSX 14974 kb)

Additional file 2: Figure S1. Reduced expression levels of twin lead to a change on the number of positive $\mathrm{pH} 3$ on eye discs from ey-Gal4 wild type and sensitized fly models. Quantification of the number of $\mathrm{pH} 3 \mathrm{posi}-$ tive cells on the posterior portion of eye-antennal imaginal discs $(n=10)$ from sensitized + twin larvae. Representative confocal images of eyeantennal imaginal discs of the indicated genotypes. Green staining: BrdU, pH3 or cleaved-DCP1a positive cells; red staining: ELAV protein. Results are compared with data of the other genotypes shown in Figs. 2c and 4a. Scale bars are $70 \mu \mathrm{M}$. (PDF $4225 \mathrm{~kb}$ )

Additional file 3 Figure S2. Expression of the different CCR4-NOT subunits after downregulation on ey-Gal4 wild-type and sensitized fly models. Downregulation of Not1, Not2, Pop2, and twin on wild-type eyeantennal discs and B) Downregulation of Not1, Not2, Pop2, and twin on sensitized eye-antennal discs. Bars represent expression mRNA levels, normalized using Rps13 as house-keeping gene and ey-Gal4 (wild-type) + UAS-RNAi white as reference sample. Each bar represents pool of 40 eyeantennal discs isolated from $40 \mathrm{~L} 3$ wandering larvae from two independent crosses. (PDF $390 \mathrm{~kb}$ )

Additional file 4: Figure S3. Similar expression levels are achieved and translated proteins are equally stable when we ectopically express the different Not3 constructs. A) The yellow graph bar represents the percentage of transfection achieved on S2 cells. Western blot analyses show protein levels of the different Not3 proteins $24 \mathrm{~h}$ after gene expression induction, with or without cycloheximide treatment. No differences on protein expression/stability among the UAS-Not constructs were observed. Asterisks indicate unspecific protein bands. B) qPCR analyses showing the Not3 expression levels on each genotype. Each bar represents the expression value of a pool of 10 individuals. (PDF 422 kb)

Additional file 5: Figure S4. Stabilization of mRNA expression levels of Cycb, fancl, and upd2 upon Not3 downregulation and transcription inhibition on Drosophila S2 cells in vitro. A) Percentage of YFP expressing cells upon addition of CuSO4 on the cell media. B) Expression of the different genes at time-point 0 . Our results show a clear knockdown of the Not3 mRNA expression (almost 50\% efficiency) at time point $0 \mathrm{~h}$, which was associated with upregulation of $(y c b$, fancl, and upd2. C) Cycb, fancl, and upd2 mRNAs showed a moderate increase in stability after Not3 downregulation when compared with the control condition (YFP expression). In all figure panels, results are shown as mean \pm S.D. Three independent experiments were performed. (PDF 418 kb)

\section{Abbreviations}

CC: Coiled coil; Cycb: Cyclin B; DI: Notch ligand Delta; dpa: Discs proliferation abnormal; GSEA: Gene set enrichment analysis; NB: Not-box; pH3: PhosphoHistone3; T-ALL: T-cell acute lymphoblastic leukemia; upd: Unpaired

\section{Acknowledgements}

The rat anti-Elav 7E8A10 antibody developed by Gerald Rubin was obtained from the Developmental Studies Hybridoma Bank. Stocks were obtained from the Bloomington Drosophila Stock Center and the Vienna Drosophila Resource Center. This study was supported by grants from the European Research Council and the Interuniversity Attraction Poles granted by the Federal Office for Scientific, Technical and Cultural Affairs, Brussels, Belgium (JC) the FWO-Vlaanderen (RS); and the European Hematology Association (CV).

\section{Funding}

This study was supported by grants from the European Research Council (JC), KU Leuven project financing SymBioSys (JC), the FWO-Vlaanderen (RS), and the European Hematology Association (CV).

\section{Availability of data and materials}

The datasets used and/or analyzed during the current study are available from the corresponding author on reasonable request. All data generated or analyzed during this study are included in this published article [and its supplementary information files]

\section{Authors' contributions}

$\mathrm{CV}, \mathrm{JC}, \mathrm{BH}, \mathrm{MA}$, and $\mathrm{GH}$ contributed to the conceptualization of the study. $\mathrm{CV}, \mathrm{RS}, \mathrm{SD}, \mathrm{OG}$, and JY contributed to the methodology and analysis. CV, RS, $\mathrm{SD}, \mathrm{JC}$, and $\mathrm{BH}$ contributed to the investigation. $\mathrm{CV}, \mathrm{RS}, \mathrm{SD}, \mathrm{BH}, \mathrm{GH}$, and JC wrote the manuscript. All authors read and approved the final manuscript.

\section{Ethics approval}

All animal experiments were carried out in accordance with the National Institutes of Health's Guidelines for the Care and Use of Laboratory Animals.

\section{Consent for publication}

This is not applicable for this study.

\section{Competing interests}

The authors declare that they have no competing interests.

\section{Publisher's Note}

Springer Nature remains neutral with regard to jurisdictional claims in published maps and institutional affiliations.

\section{Author details}

${ }^{1}$ Center for Cancer Biology, VIB, Leuven, Belgium. ${ }^{2}$ Center for Human Genetics, KU Leuven, Herestraat 49, box 912, B-3000 Leuven, Belgium. ${ }^{3}$ Department of Oncology, KU Leuven, Leuven, Belgium. ${ }^{4}$ Center for Brain \& Disease Research, VIB, Leuven, Belgium. ${ }^{5}$ Institut du Cerveau et de la Moelle Epinière (ICM) - Hôpital Pitié-Salpêtrière, UPMC, Sorbonne Universités, Inserm CNRS, Paris, France. ${ }^{6}$ Centro de Investigación Médica Aplicada, Av. de Pío XII, 55, 31008 Pamplona, Spain.

Received: 27 February 2018 Accepted: 13 August 2018

Published online: 25 August 2018

\section{References}

1. Collart MA, Panasenko OO. The Ccr4--not complex. Gene. 2012;492:42-53.

2. Shirai Y-T, Suzuki T, Morita M, Takahashi A, Yamamoto T. Multifunctional roles of the mammalian CCR4-NOT complex in physiological phenomena. Front Genet. 2014:5:286.

3. Wahle E, Winkler GS. RNA decay machines: deadenylation by the Ccr4-not and Pan2-Pan3 complexes. Biochim Biophys Acta. 1829:2013:561-70.

4. Ito K, Inoue T, Yokoyama K, Morita M, Suzuki T, Yamamoto T. CNOT2 depletion disrupts and inhibits the CCR4-NOT deadenylase complex and induces apoptotic cell death. Genes Cells. 2011;16:368-79.

5. Ito K, Takahashi A, Morita M, Suzuki T, Yamamoto T. The role of the CNOT1 subunit of the CCR4-NOT complex in mRNA deadenylation and cell viability. Protein Cell. 2011;2:755-63.

6. Morita M, Oike Y, Nagashima T, Kadomatsu T, Tabata M, Suzuki T, et al. Obesity resistance and increased hepatic expression of catabolism-related mRNAs in Cnot3+/- mice. EMBO J. 2011:30:4678-91.

7. Watanabe C, Morita M, Hayata T, Nakamoto T, Kikuguchi C, Li X, et al. Stability of mRNA influences osteoporotic bone mass via CNOT3. Proc Natl Acad Sci U S A. 2014;111:2692-7.

8. Inoue T, Morita M, Hijikata A, Fukuda-Yuzawa Y, Adachi S, Isono K, et al. CNOT3 contributes to early B cell development by controlling Igh rearrangement and p53 mRNA stability. J Exp Med. 2015;212:1465-79.

9. Mayr C, Bartel DP. Widespread shortening of $3^{\prime} U T R s$ by alternative cleavage and polyadenylation activates oncogenes in cancer cells. Cell. 2009;138:673-84.

10. Wiestner A, Tehrani M, Chiorazzi M, Wright G, Gibellini F, Nakayama K, et al. Point mutations and genomic deletions in CCND1 create stable truncated cyclin D1 mRNAs that are associated with increased proliferation rate and shorter survival. Blood. 2007;109:4599-606. 
11. Rounbehler RJ, Fallahi M, Yang C, Steeves MA, Li W, Doherty JR, et al. Tristetraprolin impairs myc-induced lymphoma and abolishes the malignant state. Cell. 2012;150:563-74

12. Hodson DJ, Janas ML, Galloway A, Bell SE, Andrews S, Li CM, et al. Deletion of the RNA-binding proteins ZFP36L1 and ZFP36L2 leads to perturbed thymic development and T Iymphoblastic leukemia. Nat Immunol. 2010;11:717-24.

13. De Keersmaecker K, Atak ZK, Li N, Vicente C, Patchett S, Girardi T, et al. Exome sequencing identifies mutation in CNOT3 and ribosomal genes RPL5 and RPL10 in T-cell acute lymphoblastic leukemia. Nat Genet. 2013;45:186-90.

14. Vicente C, Schwab C, Broux M, Geerdens E, Degryse S, Demeyer S, et al. Targeted sequencing identifies associations between IL7R-JAK mutations and epigenetic modulators in T-cell acute lymphoblastic leukemia. Haematologica. 2015;100:1301-10.

15. Seki M, Kimura S, Isobe T, Yoshida K, Ueno H, Nakajima-Takagi Y, et al. Recurrent SPI1 (PU.1) fusions in high-risk pediatric T cell acute lymphoblastic leukemia. Nat Genet. 2017;49:1274-81.

16. Liu Z, Hornakova T, Hornakova T, Li F, Staerk J, Staerk J, et al. Acute lymphoblastic leukemia-associated JAK1 mutants activate the Janus kinase/ STAT pathway via interleukin-9 receptor alpha homodimers. J Biol Chem. 2009;284:6773-81.

17. La Starza R, Barba G, Demeyer S, Pierini V, Di Giacomo D, Gianfelici V, et al. Deletions of the long arm of chromosome 5 define subgroups of T-cell acute lymphoblastic leukemia. Haematologica. 2016;101:951-8.

18. Bossuyt W, De Geest N, Aerts S, Leenaerts I, Marynen P, Hassan BA. The atonal proneural transcription factor links differentiation and tumor formation in Drosophila. PLoS Biol. 2009;7:e40.

19. Kim D, Pertea G, Trapnell C, Pimentel H, Kelley R, Salzberg SL. TopHat2: accurate alignment of transcriptomes in the presence of insertions, deletions and gene fusions. Genome Biol. 2013;14:R36.

20. Anders S, Pyl PT, Huber W. HTSeq--a Python framework to work with highthroughput sequencing data. Bioinformatics. 2015;31:166-9.

21. Love MI, Huber W, Anders S. Moderated estimation of fold change and dispersion for RNA-seq data with DESeq2. Genome Biol. 2014;15:550.

22. Lyne R, Smith R, Rutherford K, Wakeling M, Varley A, Guillier F, et al. FlyMine: an integrated database for Drosophila and Anopheles genomics. Genome Biol. 2007:8:R129.

23. Dagklis A, Pauwels D, Lahortiga I, Geerdens E, Bittoun E, Cauwelier B, et al. Hedgehog pathway mutations in T-cell acute lymphoblastic leukemia. Haematologica. 2015;100:e102-5.

24. Jiao X, Sherman BT, Huang DW, Stephens R, Baseler MW, Lane HC, et al. DAVID-WS: a stateful web service to facilitate gene/protein list analysis. Bioinformatics. 2012;28:1805-6.

25. Ferres-Marco D, Gutierrez-Garcia I, Vallejo DM, Bolivar J, Gutierrez-Aviño FJ, Dominguez M. Epigenetic silencers and Notch collaborate to promote malignant tumours by Rb silencing. Nature. 2006;439:430-6.

26. Neely GG, Kuba K, Cammarato A, Isobe K, Amann S, Zhang L, et al. A global in vivo Drosophila RNAi screen identifies NOT3 as a conserved regulator of heart function. Cell. 2010;141:142-53.

27. Basquin J, Roudko W, Rode M, Basquin C, Séraphin B, Conti E. Architecture of the nuclease module of the yeast Ccr4-not complex: the Not1-Caf1-Ccr4 interaction. Mol Cell. 2012:48:207-18.

28. Petit A-P, Wohlbold L, Bawankar P, Huntzinger E, Schmidt S, Izaurralde E, et al. The structural basis for the interaction between the CAF1 nuclease and the NOT1 scaffold of the human CCR4-NOT deadenylase complex. Nucleic Acids Res. 2012:40:11058-72

29. Hu G, Kim J, Xu Q, Leng Y, Orkin SH, Elledge SJ. A genome-wide RNAi screen identifies a new transcriptional module required for self-renewal. Genes Dev. 2009;23(7):837-48.

30. Bach EA, Ekas LA, Ayala-Camargo A, Flaherty MS, Lee H, Perrimon N, et al. GFP reporters detect the activation of the Drosophila JAK/STAT pathway in vivo. Gene Expr Patterns. 2007;7:323-31.

31. Puente XS, Beà S, Valdés-Mas R, Villamor N, Gutiérrez-Abril J, Martín-Subero $\mathrm{Jl}$, et al. Non-coding recurrent mutations in chronic lymphocytic leukaemia. Nature. 2015;526:519-24.

32. Faraji F, Hu Y, Wu G, Goldberger NE, Walker RC, Zhang J, et al. An integrated systems genetics screen reveals the transcriptional structure of inherited predisposition to metastatic disease. Genome Res. 2014;24:227-40.

33. Cejas P, Cavazza A, Yandava CN, Moreno V, Horst D, Moreno-Rubio J, et al. Transcriptional regulator CNOT3 defines an aggressive colorectal cancer subtype. Cancer Res. 2017;77:766-79.
34. Richter-Pechańska P, Kunz JB, Hof J, Zimmermann M, Rausch T, Bandapalli $\mathrm{OR}$, et al. Identification of a genetically defined ultra-high-risk group in relapsed pediatric T-lymphoblastic leukemia. Blood Cancer J. 2017;7:e523.

35. Suzuki T, Kikuguchi C, Sharma S, Sasaki T, Tokumasu M, Adachi S, et al. CNOT3 suppression promotes necroptosis by stabilizing mRNAs for cell death-inducing proteins. Sci Rep. 2015;5:14779.

36. Song Y, Zhao C, Dong L, Fu M, Xue L, Huang Z, et al. Overexpression of cyclin B1 in human esophageal squamous cell carcinoma cells induces tumor cell invasive growth and metastasis. Carcinogenesis. 2008;29:307-15.

37. Yuan J, Yan R, Krämer A, Eckerdt F, Roller M, Kaufmann M, et al. Cyclin B1 depletion inhibits proliferation and induces apoptosis in human tumor cells. Oncogene. 2004;23:5843-52

38. Kauffmann A, Rosselli F, Lazar V, Winnepenninckx V, Mansuet-Lupo A, Dessen $P$, et al. High expression of DNA repair pathways is associated with metastasis in melanoma patients. Oncogene. 2008;27:565-73.

39. Ruggero D, Pandolfi PP. Does the ribosome translate cancer? Nat Rev Cancer. 2003:3:179-92.

40. Boon K, Caron HN, van Asperen R, Valentijn L, Hermus MC, van Sluis P, et al $\mathrm{N}$-myc enhances the expression of a large set of genes functioning in ribosome biogenesis and protein synthesis. EMBO J. 2001;20:1383-93.

41. Shenoy N, Kessel R, Bhagat TD, Bhattacharyya S, Yu Y, McMahon C, et al. Alterations in the ribosomal machinery in cancer and hematologic disorders. J Hematol Oncol. 2012:5:32

42. Kim J-H, You K-R, Kim IH, Cho B-H, Kim C-Y, Kim D-G. Over-expression of the ribosomal protein L36a gene is associated with cellular proliferation in hepatocellular carcinoma. Hepatology. 2004;39:129-38.

\section{Ready to submit your research? Choose BMC and benefit from:}

- fast, convenient online submission

- thorough peer review by experienced researchers in your field

- rapid publication on acceptance

- support for research data, including large and complex data types

- gold Open Access which fosters wider collaboration and increased citations

- maximum visibility for your research: over $100 \mathrm{M}$ website views per year

At $\mathrm{BMC}$, research is always in progress.

Learn more biomedcentral.com/submissions 